Method Changes in ventilatory and haemodynamic parameters during occupational exposure to ultrafine particles summers studied using a survey-type retrospective cohort exposed unexposed conducted in a company producing agricultural equipment for a period of five month period from January 1 to May 30, 2013 in 139 subjects, including 107 exposed men and 3 unexposed.

The survey consisted of a questionnaire (WHO), a complete physical examination with measurement of blood pressure before and after the job, a spirometer before and after the job.

Results $18.7 \%$ were presented, a type of respiratory symptoms in chronic bronchitis against only $6.3 \%$ of non-exposed with a statistically significant difference $(\mathrm{P}=.04)$.

The prevalence of chronic bronchitis was significantly higher in smokers than in nonsmokers with $23.3 \%$ against $7.6 \%$ respectively. $(\mathrm{P}=.01)$.

The papers have a higher incidence of lung disease than unexposed with $83.2 \%$ of restrictive lung disease after exposure in exposed against $78.1 \%$ in the unexposed.

Smokers with normal spirometry is less than non-smokers before and after exposure with the following frequencies after exposure: $12.1 \%$ in non-smokers against only $4.1 \%$ in smokers.

On haemodynamic parameters was noticed an increase in TAP $(47.87 \mathrm{mmHg})$, FC $(76.16 \mathrm{~mm} \mathrm{Hg})$ after exposure in exposed.

Conclusions Our results have demonstrated the harmful effects of ultrafine particles on changes in ventilatory and haemodynamic parameters.

\section{SEVERITY OF ILLNESS ASSOCIATED WITH WATER RECREATION}

Stephanie DeFlorio-Barker, Samuel Dorevitch. University of Illinois at Chicago, Chicago, Illinois, USA

\subsection{6/oemed-2014-102362.227}

Objectives We evaluated the severity of illness among those engaging in limited-contact water recreation such as boating, fishing, kayaking, and rowing.

Method Data were obtained from a cohort study which assessed the development of illness following water recreation. Disease severity was defined as symptom-days, the total number of days with symptoms related to gastrointestinal illness, respiratory illness, or eye, ear, and skin symptoms. Severity was evaluated in association with the degree of water exposure. Analysis included logistic regression and G-computation.

Results 11297 participants completed the cohort study, of which 2301 developed symptoms related to gastrointestinal illness, respiratory illness, or eye, ear, or skin infection. When evaluating both ill and healthy participants who participated in water recreation, total symptom-days ranged from 0-67, and exhibited a right-skewed distribution. When dichotomized at the $90^{\text {th }}$ percentile, there was a crude relative risk (RR) of 1.47 (1.27-1.72) for those getting their face wet during water recreation, and a RR of $1.65(1.28-2.12)$ for those indicating that they swallowed water during water recreation.

Conclusions Increased water exposure, resulting in getting the face wet, or swallowing water is related to increased disease severity among water recreators. Further analysis is necessary to determine if any covariates such as age, race/ethnicity, gender, or previous comorbidities modify or confound the relationship between water exposure and disease severity.
0101 WORK HOURS, JOB STRAIN, AND OCCUPATION WITH ENDOTHELIAL FUNCTION: THE MULTI-ETHNIC STUDY OF ATHEROSCLEROSIS (MESA)

${ }^{1}$ Luenda Charles, ${ }^{1}$ Desta Fekedulegn, ${ }^{2}$ Paul Landsbergis, ${ }^{1}$ Cecil Burchfiel, ${ }^{3}$ Sherry Baron, ${ }^{4} J o e l$ Kaufman, ${ }^{5}$ Karen Hinckley Stukovsky, ${ }^{3}$ Kaori Fujishiro, ${ }^{6}$ Capri Foy, ${ }^{1}$ Michael Andrew, ${ }^{7}$ Ana Diez Roux. 'Biostatistics and Epidemiology Branch, Health Effects Laboratory Division, National Institute for Occupational Safety and Health, Morgantown, WV, USA; ${ }^{2}$ Department of Environmental and Occupational Health Sciences, State University of New York Downstate Medical Center and State University of New York Downstate School of Public Health, Brooklyn, NY, USA; ${ }^{3}$ Division of Surveillance, Hazard Evaluation, and Field Studies, National Institute for Occupational Safety and Health, Cincinnati, $\mathrm{OH}$, USA; ${ }^{4}$ Department of Environmental and Occupational Health Sciences, School of Public Health, University of Washington, Seattle, WA, USA; ${ }^{5}$ Department of Biostatistics, School of Public Health, University of Washington, Seattle, WA, USA; ${ }^{6}$ Department of Social Sciences and Health Policy, Division of Public Health Sciences, Wake Forest School of Medicine, Winston-Salem, NC, USA; 'Department of Epidemiology, School of Public Health, University of Michigan, Ann Arbor, MI, USA

10.1136/oemed-2014-102362.228

Objectives To investigate associations of work hours, job control, job demands, job strain, and occupational category with endothelial function, a predictor of cardiovascular disease (CVD).

Method Currently employed participants free of CVD ( $\mathrm{n}=$ 1499; 55.5\% men; 62\% non-white) of the Multi-Ethnic Study of Atherosclerosis provided information on work hours, job decision latitude, and job demands. Responses to current occupation were coded using the Census 2000 Occupational Codes; codes were collapsed to provide four occupational categories. Brachial artery flow-mediated dilation (FMD), a validated measure of endothelial function, was obtained using high-resolution ultrasound. Mean values of FMD, expressed as percent change in brachial artery diameter, were examined across categories of work hours $(<40,40,41-49, \geq 50)$ and the other exposures using ANOVA/ANCOVA.

Results Occupational category was significantly associated with FMD overall, with Blue-collar workers showing the lowest mean values after adjustment for age, gender, race/ethnicity, education, waist circumference, total and HDL cholesterol, body mass index, systolic and diastolic blood pressure, physical activity, smoking status, and pack-years of smoking: Management/Professional $=4.96 \pm 0.22 \%$; Sales $/$ Office $=5.06 \pm 0.27 \%$; Services $=4.70 \pm 0.29 \%$; Blue-collar workers $=4.18 \pm 0.27 \%$ (adjusted $\mathrm{p}=0.001)$. There was evidence of effect modification between occupational category and FMD by gender $(p=0.031)$ such that in stratified analyses, significant associations were observed among women (adjusted $\mathrm{p}=0.002$ ) but not men (adjusted $\mathrm{p}=0.098$ ). None of the other work exposures were significantly associated with FMD.

Conclusions Blue-collar workers had decreased endothelial function compared to other workers; potential reasons should be examined in future studies. Decreased endothelial function may reflect a biological mechanism explaining occupational differences in CVD.

\section{A RETROSPECTIVE COHORT MORTALITY STUDY OF US PHOSPHATE INDUSTRY WORKERS: AN UPDATE}

James Yiin, Travis Kubale, Robert Daniels, Kevin Dunn. National Institute for Occupational Safety and Health, Cincinnati, OH, USA

10.1136/oemed-2014-102362.229 